\title{
Dispersion relation of electromagnetic ion cyclotron waves using Cluster observations
}

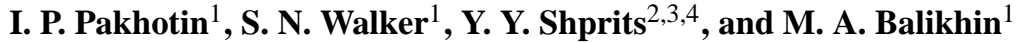 \\ ${ }^{1}$ Department of Automatic Control and Systems Engineering, University of Sheffield, Sheffield, South Yorkshire, UK \\ ${ }^{2}$ Department of Earth and Space Sciences, UCLA, Los Angeles, CA, USA \\ ${ }^{3}$ Department of Atmospheric and Planetary Sciences, MIT, Cambridge, MA, USA \\ ${ }^{4}$ Skolkovo Institute of Science and Technology, Moscow, Russian Federation
}

Correspondence to: I. P. Pakhotin (mea07ip@ sheffield.ac.uk)

Received: 14 January 2013 - Revised: 5 July 2013 - Accepted: 18 July 2013 - Published: 26 August 2013

\begin{abstract}
Multi-point wave observations on Cluster spacecraft are used to infer the dispersion relation of electromagnetic ion cyclotron (EMIC) waves. In this study we use a phase differencing method and observations from STAFF and WHISPER during a well-studied event of 30 March 2002. The phase differencing method requires the knowledge of the direction of the wave vector, which was obtained using minimum variance analysis. Wave vector amplitudes were calculated for a number of frequencies to infer the dispersion relation experimentally. The obtained dispersion relation is largely consistent with the cold plasma dispersion relation. The presented method allows inferring the dispersion relation experimentally. It can be also used in the future to analyse the hot plasma dispersion relation of waves near the local gyrofrequency that can occur under high plasma beta conditions.
\end{abstract}

Keywords. Magnetospheric physics (plasmasphere; plasma waves and instabilities)

\section{Introduction}

The near-Earth radiation belt environment presents a number of hazards to orbiting spacecraft (Baker, 1996). A better understanding of the nature of the magnetosphere dynamics and associated processes will in turn allow a better understanding of these dangers, allowing engineers to design spacecraft to withstand such effects. Understanding and modelling radiation belt dynamics helps mitigate spacecraft damage.

The Van Allen radiation belts are formed from energetic charged particles that are trapped by the Earth's magnetic field. In the inner magnetosphere, there are broadly two zones of enhanced trapped particle flux. The inner and outer belts are separated by a slot region at around $2-3 R_{\mathrm{E}}$ where energetic particle flux levels are usually low. The existence of the slot region is a result of the balance between an inward radial diffusion of electrons from the outer belt and precipitation losses due to resonant scattering by very low frequency (VLF) waves, primarily plasmaspheric hiss (e.g. Lyons et al., 1972; Lyons and Thorne, 1973; Selesnick et al., 2003; Thorne et al., 2007; Shprits et al., 2008b). The fluxes of high energy particles in the inner belt are relatively stable, with a predictable variation linked to the solar cycle ( $\mathrm{Li}$ et al., 2001). However, the relativistic electron fluxes in the outer belt are highly variable, and can change over an order of magnitude in a few hours (e.g. Thorne et al., 2007).

Radiation belt enhancements appear to be correlated with geomagnetic storm activity, but the link between storm activity and radiation belt enhancement is complicated, with relativistic electron flux increases observed only in about half of the cases studied (Reeves et al., 2003). One-fifth of all storms analysed produced flux decreases, with the rest leaving flux levels largely unchanged. Clearly, several competing processes exist during times of enhanced geomagnetic activity that produce both particle acceleration and loss. In their review, Friedel et al. (2002) identified several mechanisms for the build-up of energetic $(\mathrm{MeV})$ electrons in the magnetosphere. Most sources listed in the review fall broadly into two categories: those which rely on inward radial diffusion (Kellogg, 1959; Tverskoy, 1964, 1965; Fälthammar, 1965; Elkington et al., 2003), and those with a local acceleration mechanism. Radial diffusion can be enhanced by 
magnetospheric pulsations (e.g. Thorne, 2010) which are generated by large-scale disturbances such as the KelvinHelmholtz instability on the flanks of the magnetosphere (Shprits et al., 2008a, and references therein), buffeting of the magnetosphere on the day side, or by convective injections of protons on the night side. Evidence for local acceleration as a source of relativistic electrons was found by storm modelling (Summers et al., 2002). Later, Chen et al. (2006) conducted an analysis of the radial profiles of phase space density (PSD) distributions of energetic electrons using combined satellite measurements and found evidence for both the external (radial transport) and internal (wave-particle interactions) processes acting simultaneously. The study established a peak in the radial PSD profile inside the outer radiation belt, consistent with a local acceleration source. Further studies (Shprits et al., 2007) demonstrated the importance of the local stochastic acceleration of particles by their interaction with VLF waves, in particular chorus waves, during magnetic storm recovery.

Wave-particle interactions can transfer energy from one part of the energetic particle distribution to another, resulting in local particle acceleration. Change of the pitch angle resulting from wave-particle interactions can lead to the precipitation via the loss cone. Thus plasma waves can cause both flux enhancement and depletion.

Electromagnetic ion cyclotron (EMIC) waves are excited in the Pc 1-2 range (period 0.2-10 s) just below each plasma ion species gyrofrequency usually in the region of the magnetic equator (e.g. Fraser et al., 1996). It is commonly accepted that these waves are excited by anisotropic ring current ions injected into the inner magnetosphere during substorms (Summers and Thorne, 2003; Li et al., 2007) or by aforementioned changes in the plasma distribution function produced by lower frequency waves (e.g. Fraser et al., 1992; Thorne, 2010). EMIC waves propagate and grow around the dense plasmasphere region near the plasmapause (Fraser and Nguyen, 2001), cyclotron-resonate with radiation belt ions and Landau-resonate with outer radiation belt electrons (Horne and Thorne, 1990, 1994) scattering both species into the loss cone. Thus they are believed responsible for both radiation belt depletions and ring current ion losses (e.g. Fraser et al., 2010). They provide possibly the dominant mechanism for scattering electrons into the loss cone in the inner magnetosphere (Shprits et al., 2008b). While there are many reports of EMIC waves observations based on the spectra of observed turbulence, this study is devoted to the determination of the dispersion relation of EMIC waves directly from multi-point Cluster observations.

Since the initial analytical work (Stix, 1962; Smith and Brice, 1964) identifying the dispersion surfaces for ion cyclotron waves, there have been numerous theoretical works on ion cyclotron dispersion relations. It is now known that significant numbers of heavy ions such as $\mathrm{He}^{+}$and $\mathrm{O}^{+}$exist in the inner magnetosphere, and so the dispersion relations for multiple-ion plasmas in the presence of heavy ions have been theoretically calculated (Smith and Brice, 1964; Young et al., 1981; Swanson, 1989; Horne and Thorne, 1990). However, there has been relatively little experimental work to confirm the theory and ray tracing simulations directly. Broughton et al. (2008) used the wave telescope method to analyse ultra-low frequency (ULF) waves in the plasma sheet boundary layer. This paper uses the cospectral/phase differencing method of $\boldsymbol{k}$ vector determination (Balikhin and Gedalin, 1993; Dudok de Wit et al., 1995; Balikhin et al., 1997a) to calculate the frequency-wavenumber relation directly and then compare it to existing EMIC wave dispersion theory. This method assumes that in the plasma rest frame (PF) a wave field can be represented as $\boldsymbol{B}(\boldsymbol{r}, t)=$ $\Sigma_{\omega} \boldsymbol{B}(\omega) \exp j(\boldsymbol{k r}-\omega t)+C$, where $C$ is the complex conjugate term. The PF frequency $\omega$ and the wave vector $\boldsymbol{k}$ are related by the dispersion relation $\omega=\omega(\boldsymbol{k})$. If two satellites are separated by a vector $\boldsymbol{R}$, the phase shift $\Delta \psi$ between the two measured time series at the observed frequency $\omega_{1}$ is estimated as

$\Delta \psi\left(\omega_{1}\right)=\left(\boldsymbol{k}\left(\omega_{1}\right) \boldsymbol{R}\right)=\left|k\left(\omega_{1}\right)\right||R| \cos \left(\theta_{k R}\right)$.

Thus the phase shift is a function of the scalar product of $\boldsymbol{k}$ and $\boldsymbol{R}$. The result is a projection of $\boldsymbol{k}$ on $\boldsymbol{R}$; to calculate the full $\boldsymbol{k}$ vector, knowledge of its direction is required. In the case of magnetic field measurements, the minimum variance method (Sonnerup and Cahill, 1967) can be applied to identify the direction of the wave vector. This method has the limitation that it can only be used for circularly/elliptically polarised waves. Since EMIC waves are often left-hand circular polarised at generation (e.g. Young et al., 1981), minimum variance can be exploited in this study. Finally, knowledge of the wave vector for any observed frequency $\omega_{1}$ "enables the determination of the wave dispersion using the Doppler relation: $\omega_{1}=\omega+\boldsymbol{k} \boldsymbol{V}_{0}\left(\boldsymbol{V}_{0}\right.$ is the plasma bulk velocity)" (Balikhin et al., 1997a). The phase differencing techniques have been exploited and enabled identification of wave modes in various regions of geospace such as foreshock, shock front and the magnetosheath (Balikhin et al., 1997b; Chisham et al., 1999; Walker et al., 2004).

\section{Instrumentation}

The Cluster satellites were launched in 2000 into a polar orbit with period of $57 \mathrm{~h}$, apogee outside the bow shock or deep in the magnetotail (depending on the season) and perigee in the inner magnetosphere. The mission consists of 4 spacecraft flying in a tetrahedral formation, allowing simultaneous multi-satellite measurements to resolve spatial and temporal effects. The Cluster fluxgate magnetometer (FGM) instrument (Balogh et al., 2001) provides measurements of magnetic fluctuations for the events studied in the present paper at a sampling frequency of $22 \mathrm{~Hz}$, making it suitable for EMIC wave analysis. The Cluster ion spectrometry (CIS) instrument (Rème et al., 2001) provides measurements of 


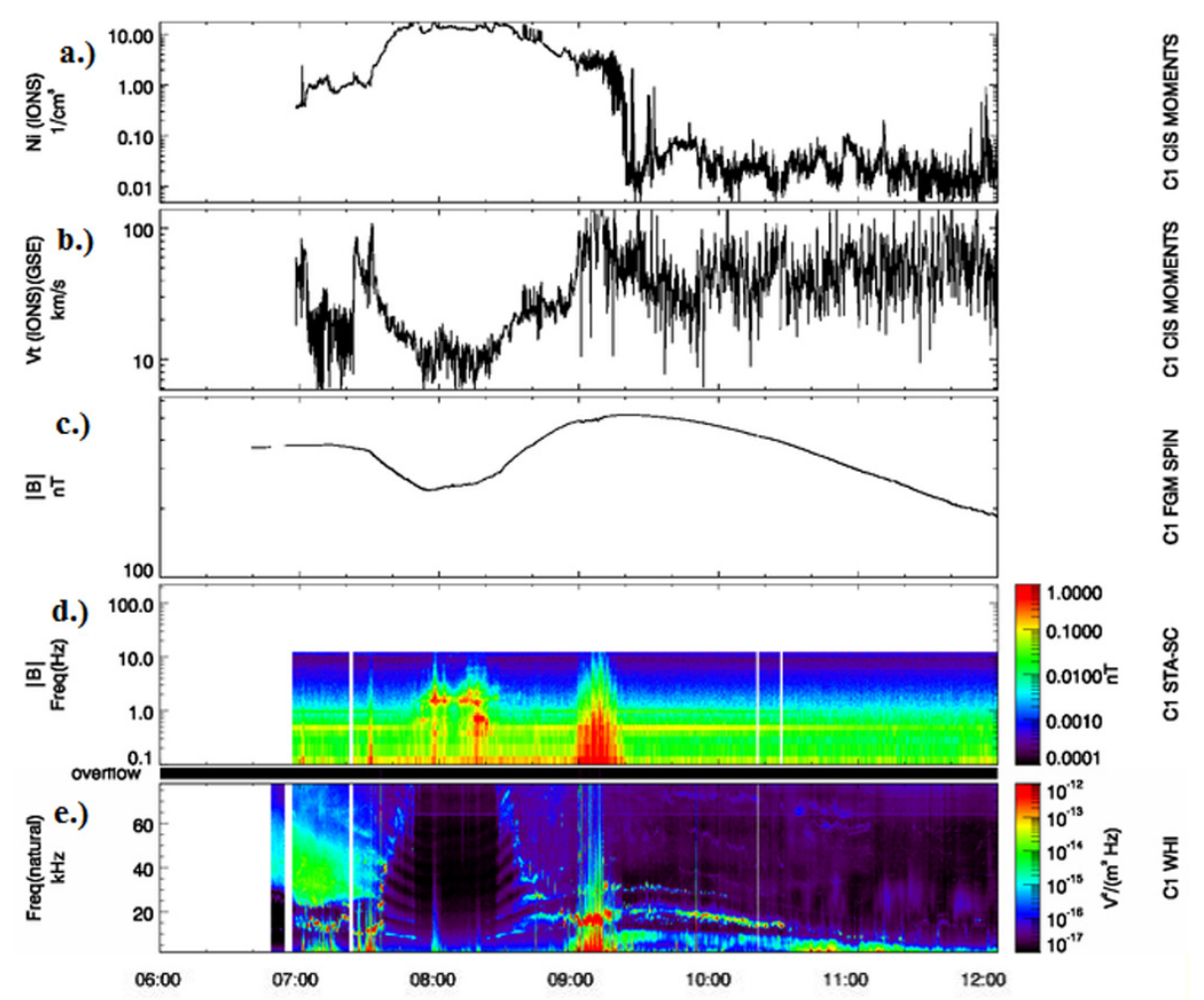

Fig. 1. Plasmapause crossing on 30 March 2002 as viewed from Cluster 1. Graphs, from top to bottom, show (a) energetic ion density recorded by CIS, (b) ion velocity from CIS, (c) magnetic field strength from FGM, (c) magnetic field waveform spectra from STAFF and (d) electric field waveform spectra from WHISPER. The inbound crossing is at 07:30, and the outbound crossing is at 08:45 UTC. EMIC waves can be seen on STAFF, the fourth panel from 08:00 until 08:30 UTC.

ion moments (mass, density, velocity, temperature parallel and perpendicular to the local magnetic field) for several ion species. The STAFF search coil magnetometers (CornilleauWehrlin et al., 2003) provide waveform data of the magnetic field sampled at $25 \mathrm{~Hz}$. WHISPER (Décréau et al., 1997) measures the electric field up to $80 \mathrm{kHz}$ in active and passive modes. The STAFF and WHISPER instruments form part of the Wave Experiment Consortium (WEC) complex that is controlled by Digital Wave Processing experiment (DWP) (Woolliscroft et al., 1997).

\section{Dispersion determination methodology}

Time series data or Morlet wavelet spectral plots are analysed for all four satellites for the duration of an event. Individual wave packets are identified by the shape of their envelope. By visual inspection it can in some cases be determined with certainty that two wave packets observed on different satellites are in fact the same wave packet propagating from one satellite to another. In these cases a histogram of the phase difference as a function of frequency is calculated using the Morlet wavelet transform. Each wave packet shows up as a discrete frequency with an associated wave vector magnitude, which is simply $\Delta \psi /|\boldsymbol{R}|$. There is a $2 \pi$ ambiguity which is due to a periodicity in phase difference and should strictly speaking extend to $\pm \infty$. The correct $\Delta \psi$ branch can be identified from either time series data or dynamical spectrum plots, by observing which satellite first sees the wave power rise over the noise level. For each wave packet, the spectrum maximum represents a point on the dispersion relation graph. The procedure is repeated for all identifiable wave packets to obtain a set of points, which are then plotted onto a frequency-wavenumber graph. Doppler effects should be taken into account, but in the case of the event studied in this paper, the plasma velocity in the plasmasphere is about an order of magnitude lower than the wave velocity. Doppler effects are therefore insignificant.

Each wavenumber obtained in this manner is not the wavenumber in the strict sense. Rather, it is the magnitude of a vector projection of the $\boldsymbol{k}$ vector onto the inter-satellite separation vector $\boldsymbol{R}$. To determine the full $\boldsymbol{k}$ vector, knowledge of the wave direction is required. The wave vector direction is obtained using minimum variance on the left-hand circularly polarised (LHCP) wave packets (Sonnerup and Cahill, 1967). Minimum variance analysis makes use of the Maxwell equation that states that magnetic fields have zero divergence. Thus the direction where the magnetic field varies least is assumed to be the direction of the wave vector. The true wave 
vector is then calculated as $|\boldsymbol{k}|=(\Delta \psi /|\boldsymbol{R}|) / \theta_{k R}$, where $\theta_{k R}$ is the angle between the wave vector and the separation vector.

\section{Cluster observation of EMIC waves on 30 March 2002}

The emissions under study took place between 08:00:50 and 08:16:30 UT. Figure 1 displays an overview plot of (a) the energetic ion density, (b) ion velocity, (c) the magnitude of the magnetic field, (d) dynamic spectrum of the magnetic field (STAFF), and (e) the dynamic spectrum of the electric field obtained by WHISPER, as observed by Cluster 1 spacecraft during time period 06:00-12:00 UT on 30 March 2002. During this time interval the Cluster spacecraft entered the plasmasphere around 07:30 UT. This can be seen implicitly by the sudden drop in plasma velocity and a rise in energetic ion density. The magnetic field dips as the spacecraft approaches the equator, then rises again after the equatorial crossing as the spacecraft moves towards higher latitudes with stronger magnetic field strength. The inbound plasmapause crossing can also be clearly identified by the rise in the electron plasma frequency seen by WHISPER until it is out of range of the instrument. The outbound crossing can be seen at 08:45 UT as indicated by a corresponding rapid drop in electron plasma frequency. Multiple EMIC waves were encountered while the spacecraft were inside the plasmasphere. The EMIC waves can be seen on the STAFF dynamic spectrum while the spacecraft were inside the plasmapause as enhancements in the wave power in the $2-3 \mathrm{~Hz}$ range. During the time period of interest, the average separation vector between $\mathrm{C} 2$ and $\mathrm{C} 4$ was $[-4,26,-83] \mathrm{km}$, between $\mathrm{C} 1$ and $\mathrm{C} 2[-12,47,183] \mathrm{km}$ and between $\mathrm{C} 1$ and $\mathrm{C} 4[-16$, $73,100] \mathrm{km}$ in the geocentric solar ecliptic (GSE) reference frame.

The four Cluster spacecraft crossed the magnetic equator at about 08:05 UT on 30 March 2002 at $L \approx 4.3$ (Pickett et al., 2010). The emissions were observed in the dusk/premidnight time sector (22:15 MLT) where there is a rather low probability of EMIC instabilities at these $L$ values (e.g. Anderson et al., 1992; Meredith et al., 2003). The event is welldocumented in previous research (Pickett et al., 2010; Omura et al., 2010; Shoji et al., 2011; Grison et al., 2013). A polarisation analysis (Pickett et al., 2010) showed the occurrence of mostly left-hand circularly polarised (LHCP) waves of distinct frequencies separated by spectral stop bands - a typical feature of EMIC waves observed in the post-noon sector (e.g. Young et al., 1981; Horne and Thorne, 1994). Figure 2 shows a Morlet wavelet spectrogram from FGM on Cluster 1 for the time period 08:00-08:20, with wave energy first seen at around $1.5 \mathrm{~Hz}$ rising to about $3 \mathrm{~Hz}$, and about $12 \mathrm{~min}$ into the event, energy below $\approx 1 \mathrm{~Hz}$ (the local helium ion gyrofrequency $\Omega_{\mathrm{He}^{+}}$) with reduced energy at the gyrofrequency itself.
The time series of magnetic field data of all 4 satellites were analysed, to identify the time intervals during which the same wave packets were observed simultaneously by at least two spacecraft so the phase differencing methodology could be used to calculate the magnitude of the $\boldsymbol{k}$ vector. Figure 3 shows one example of magnetic waveforms for a series of wave packets. It is clear from the amplitude envelopes of some of these wave packets (as highlighted by the black box) that the same waves are observed on satellites $\mathrm{C} 1$ and $\mathrm{C} 4$. The phase shift for each wave packet is visually noticeable and corresponds to the propagation delay from one satellite to the other. In total, 21 such wave packets were analysed. Since the satellites were separated mainly along the $z$ axis in the GSE reference frame, the mostly field-aligned equatorial wave packets were usually observed to propagate with a small angle $\left(<15^{\circ}\right)$ between the wave vector and separation vector $\theta_{k R}$. This meant that there was a clearly identifiable phase difference $\Delta \psi$ (Fig. 4). Equation (1) was applied to calculate the projection of the $\boldsymbol{k}$ vector on $\boldsymbol{R}$.

The wave vector was projected onto the magnetic field vector to calculate the angle $\theta_{B k}$. The results indicated mostly field-aligned propagation, as was expected of the equatorial EMIC waves. Figure 5 shows a hodogram from the same wave packet as highlighted in Fig. 3. For easier viewing of the hodogram, only a segment of the original wave packet was used. The hodogram is obtained by transforming the magnetic field time series from GSE coordinates onto the variance frame, and allows the determination of wave polarisation. The circular polarisation is clearly visible in the bottom panel where the direction of minimum variance is directed out of the page. All wave packets used in this study were found to be left-hand circularly polarised. The method employed by Fowler et al. (1967) was used to confirm polarisation further. After about 08:15 UTC, linearly polarised wave packets appeared as the constellation moved away from the magnetic equator, as predicted by theory and previous observations (e.g. Young et al., 1981; Horne and Thorne, 1994). Minimum variance on the linear wave packets was not performed due to the limitations of the (Sonnerup and Cahill, 1967) method mentioned earlier. In theory it would have been possible to use the minimum variance free method described by Balikhin et al. (2000) to calculate the full $\boldsymbol{k}$ vector. However, this method requires the same wave packet to be observed on 4 satellites. Unfortunately, none of the wave packets identified were observed on all 4 spacecraft simultaneously.

For the 14 circularly polarised wave packets (listed in Table 1), the relation between frequency and wave vector is summarised in Fig. 6. The $x$ axis, labelled $\mathrm{K}$, corresponds to the wave vector magnitude $|\boldsymbol{k}|$ multiplied by the proton inertial length $\frac{c}{\omega_{\mathrm{pi}}}$, while the $y$ axis, labelled $\mathrm{X}$, is the wave frequency normalised by the proton gyrofrequency $\Omega_{\mathrm{H}^{+}}$. Conventions are taken from Young et al. (1981). Following the methodology of Omura et al. (2010), the plasma composition 


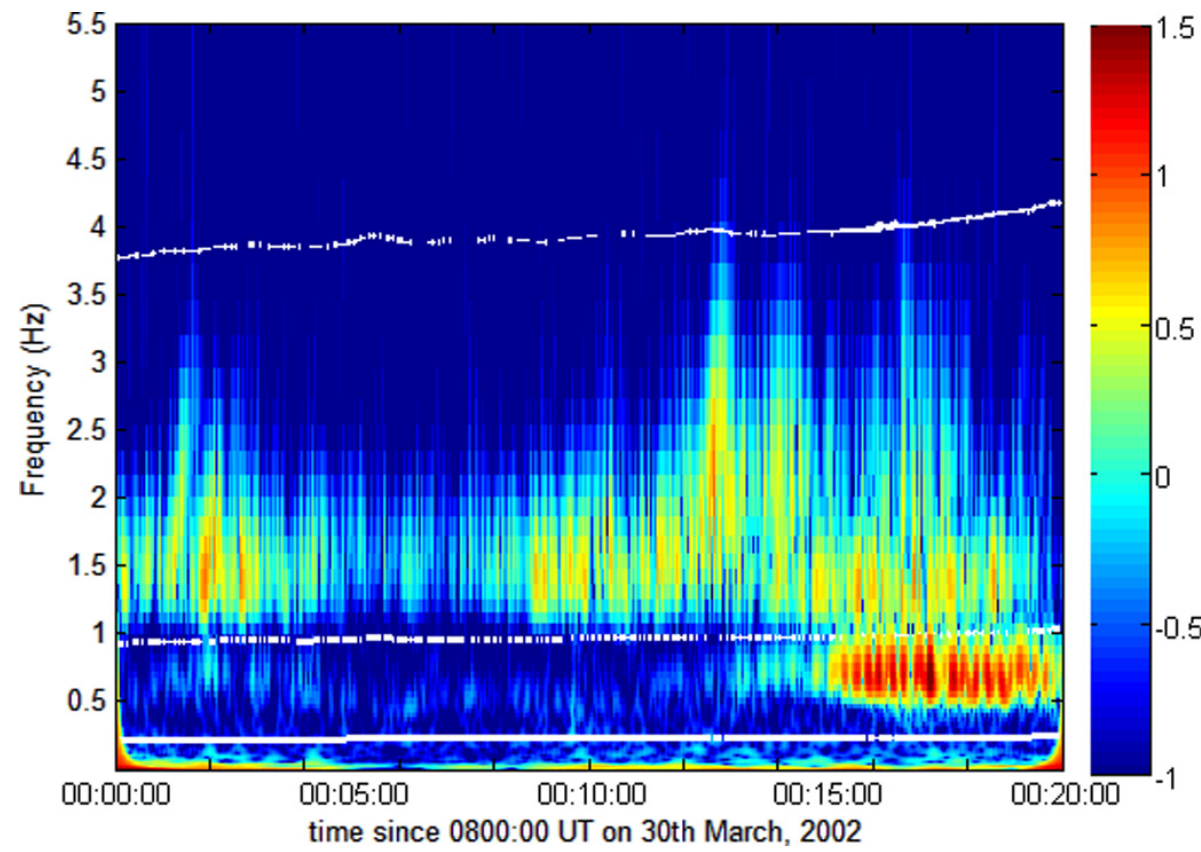

Fig. 2. Frequency-time wavelet spectrogram of ULF waves observed on 30 March 2002, on Cluster 1 ( $B_{y}$ component of magnetic field) from FGM. The colour bar is in arbitrary units representative of relative power spectral density. An area of reduced wave energy can be seen around $1 \mathrm{~Hz}$ at the local $\Omega_{\mathrm{He}^{+}}$corresponding to a stop band. The white lines, from top to bottom, represent $\Omega_{\mathrm{H}^{+}}, \Omega_{\mathrm{He}^{+}}$and $\Omega_{\mathrm{O}^{+}}$ respectively.

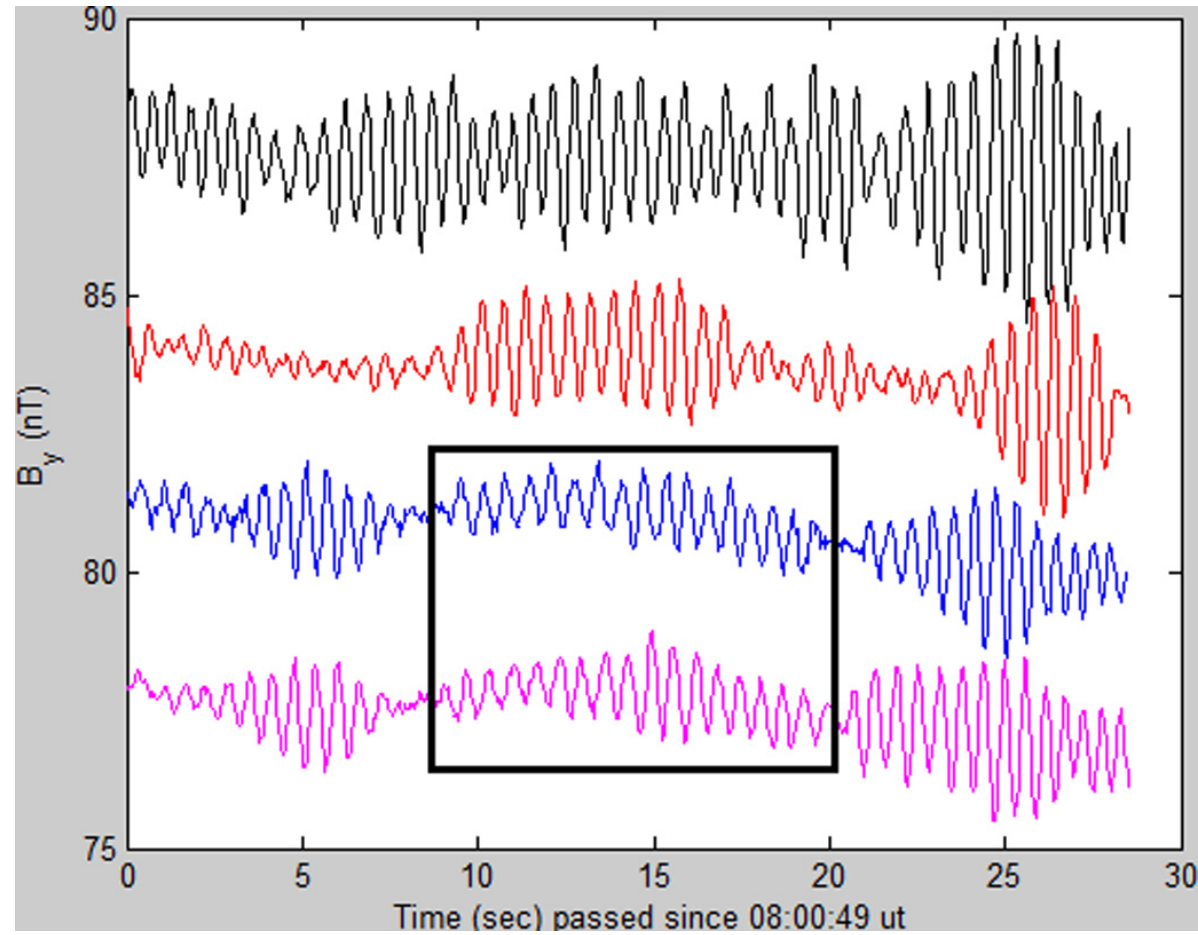

Fig. 3. Time series data of fluxgate magnetometer (FGM) instrument on board Cluster 1 (blue), Cluster 2 (red), Cluster 3 (black) and Cluster 4 (magenta) showing wave packets $49 \mathrm{~s}$ after 08:00 UTC. $B_{y}$ axis shifted $+5 \mathrm{nT}$ for Cluster 3 and $-5 \mathrm{nT}$ for Cluster 4 for better viewing. The black rectangle is around the 2 simultaneous measurements which were compared. 


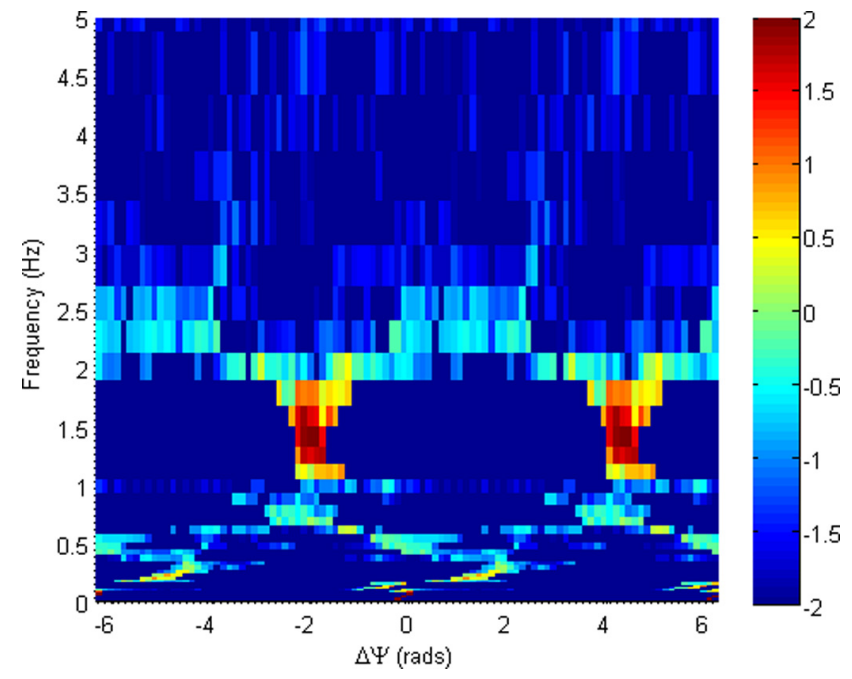

Fig. 4. The joint frequency- $\Delta \psi$ spectrum of the $B_{y}$ component of the magnetic field, for the same wave packets as pictured in Fig. 3. The colour bar is in arbitrary units representative of the relative power spectral density as a function of frequency and phase difference. The periodicity of the spectrum corresponds to an ambiguity of $2 \pi n$ in the phase difference.

was assumed to be as follows: $n_{\mathrm{e}}=178 / \mathrm{cc}, n_{\mathrm{H}^{+}}=144 / \mathrm{cc}$, $n_{\mathrm{He}^{+}}=17 / \mathrm{cc}, n_{\mathrm{O}^{+}}=17 / \mathrm{cc}$. A very similar composition, but involving energetic protons, was assumed by Shoji et al. (2011) in a simulation involving the same time period as the present study. The magnetic field magnitude averaged over the time period 08:00:50-08:16:30 and all 4 spacecraft was $253 \mathrm{nT}$.

The theoretical cold plasma dispersion is taken from the equation for the $L$ mode (Stix, 1962), which corresponds to field-aligned, left-hand polarised propagation:

$N^{2}=L=1-\sum_{i} \frac{\omega_{\mathrm{pi}}^{2}}{\omega^{2}}\left(\frac{\omega}{\omega-\epsilon_{i} \Omega_{i}}\right)$.

In Eq. (2), $N^{2}$ is the refractive index $\left(N^{2}=k^{2} c^{2} / \omega^{2}\right), \omega_{\text {pi }}$ the plasma frequency of the charged particle species $i, \Omega_{i}$ the cyclotron frequency of the charged particle species $i$, and summation is over the electrons and each positive ion species present in the plasma. $\epsilon_{i}$ is the charge state and is -1 for electrons and +1 for each ion species. In the calculation of the cold plasma dispersion relation, the plasma was assumed to contain only three species of ions $\left(\mathrm{H}^{+}, \mathrm{He}^{+}\right.$and $\left.\mathrm{O}^{+}\right)$. The resulting theoretical dispersion surface branches can be seen in Fig. 6 as solid curves. From Eq. (2) is can be seen that in a multi-ion plasma a resonance will exist at each positive ion species gyrofrequency. At these resonance points, the the refractive index and wave vector will approach infinity. The plotted analytical dispersion curves reflect this.
Table 1. Cluster satellite pairs, starting and ending times for each wave packet. Often segments of the wave packets were used in the analysis, rather than whole wave packets. This was done where noise or other waves contaminated sections of the wave packet time series. In practice, only a few oscillations are necessary to determine vector direction from minimum variance analysis.

\begin{tabular}{llll}
\hline Sat 1 & Sat 2 & Start time & End time \\
\hline C1 & C4 & $08: 00: 58$ & $08: 01: 05$ \\
C1 & C4 & $08: 01: 12$ & $08: 01: 17$ \\
C1 & C2 & $08: 08: 41$ & $08: 08: 43$ \\
C1 & C2 & $08: 08: 45$ & $08: 08: 48$ \\
C2 & C4 & $08: 12: 17$ & $08: 12: 19$ \\
C2 & C4 & $08: 12: 26$ & $08: 12: 29$ \\
C2 & C4 & $08: 12: 30$ & $08: 12: 33$ \\
C2 & C4 & $08: 12: 33$ & $08: 12: 36$ \\
C2 & C4 & $08: 12: 40$ & $08: 12: 44$ \\
C2 & C4 & $08: 13: 08$ & $08: 13: 12$ \\
C2 & C4 & $08: 13: 12$ & $08: 13: 15$ \\
C2 & C4 & $08: 15: 43$ & $08: 15: 51$ \\
C2 & C4 & $08: 15: 57$ & $08: 16: 05$ \\
C2 & C4 & $08: 16: 16$ & $08: 16: 25$ \\
\hline
\end{tabular}

\section{Discussion and conclusions}

The points in Fig. 6 in general follow the theoretical dispersion curves for cold plasma with the assumed ion composition. Two branches are evident, above and below the helium ion gyrofrequency. As the dispersion surface approaches the $\mathrm{H}^{+}$resonance, the normalised $|\boldsymbol{k}|$ approaches infinity as $\frac{\omega}{\Omega_{\mathrm{H}^{+}}}$ approaches unity. In practice collecting data near this point may be difficult: as the wavenumber approaches infinity, the phase velocity approaches zero and so the same wave packet may be seen on two satellites with a significant delay, making matching and phase difference calculations inaccurate. The results in Fig. 6 correspond well with the cold plasma approximation where left-handed waves cannot propagate in the stop band; this is also confirmed by the FGM data (Fig. 2).

Potential limitations of the methodology are summarised as follows. The cold plasma dispersion relation as outlined in Stix (1962) assumes completely field-aligned wave propagation. The phase differencing method employing wavelet transforms generates an area of high signal in frequency/phase space from which the maximum is taken as the data point value. It is possible that the true phase/frequency values do not correspond to that maximum. Plasma composition calculations are only estimations based on the cut-off frequency and cyclotron frequencies. The method used by Omura et al. (2010) assumes that helium and oxygen ions exist in equal proportions in the plasma. This is required to make the algebra a system of two equations with two unknowns. While CIS data show that energetic oxygen and helium ions do exist in similar proportions, total oxygen and 


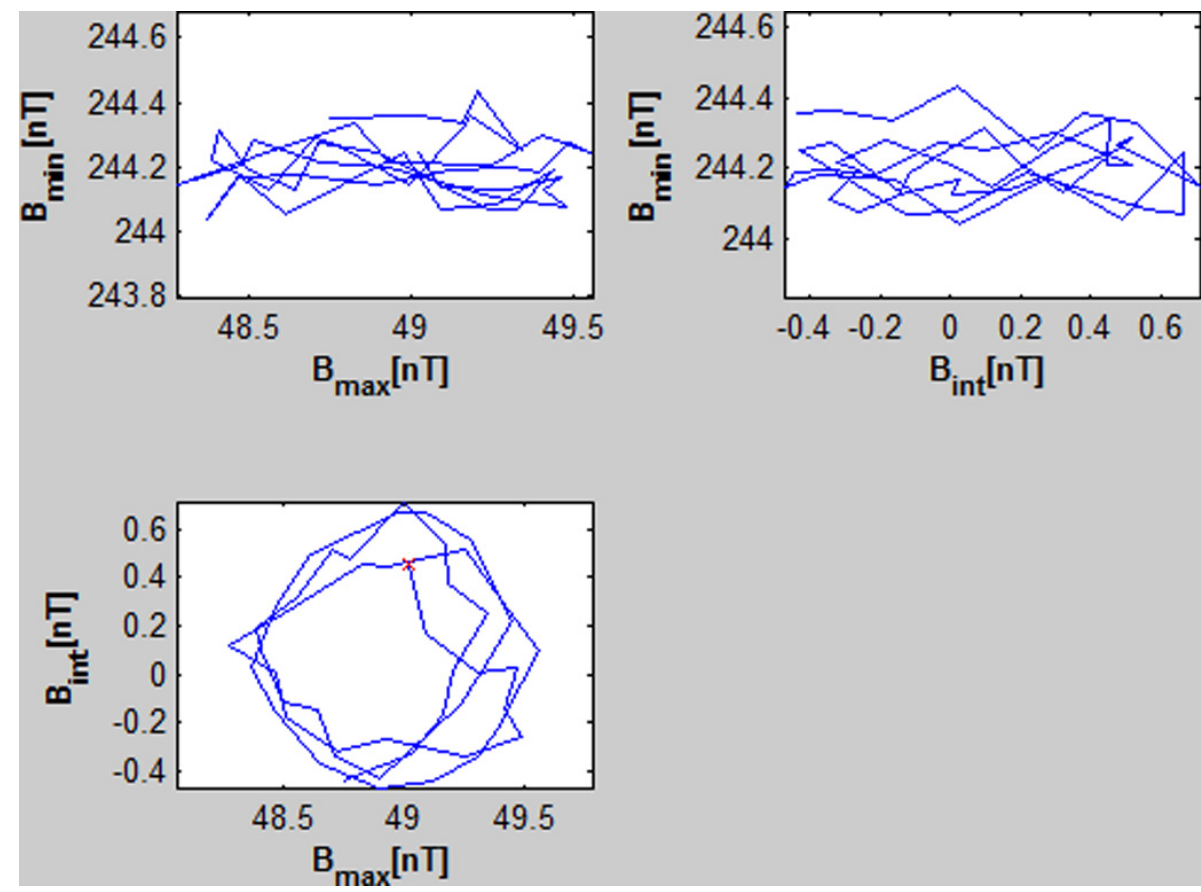

Fig. 5. Hodogram representation of a fragment of the same wave packet as in Fig. 3 seen from the Cluster 4 fluxgate magnetometer data. $B_{\max }, B_{\text {int }}$ and $B_{\min }$ signify maximum, intermediate and minimum variance eigenvectors respectively. The bottom panel shows the wave projected along the axis of minimum variance (coming out of the page). The red cross marks the beginning of the time series. Left-hand circular polarisation is clearly visible.

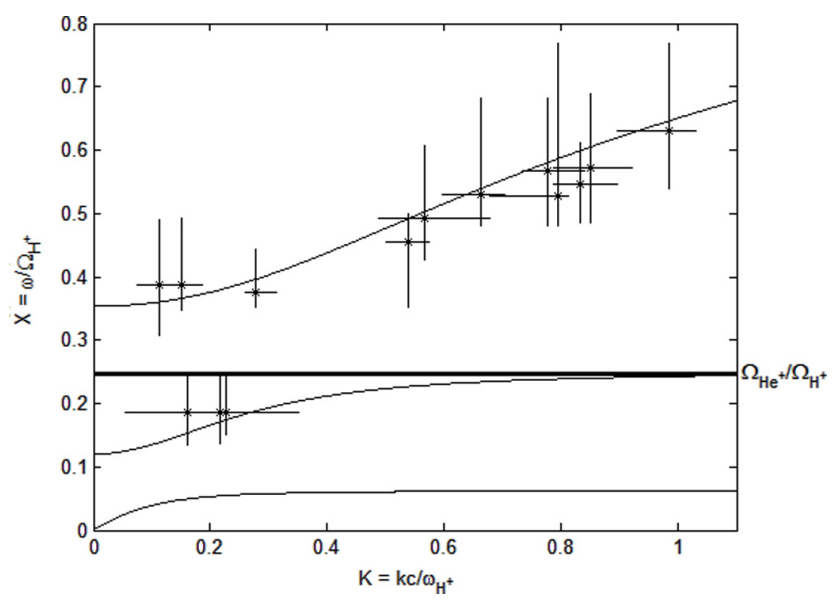

Fig. 6. Frequency vs. wavenumber relation plot of wave packets observed during the event. The bold line marks the helium ion gyrofrequency. The crosses signify individual wave packet observations overplotted with error bars in frequency and wavenumber. The solid curves are different branches of the cold plasma dispersion relation for the aforementioned plasma composition.

helium ion numbers are not necessarily equal. Assuming the cold plasma approximation at all ignores the role played by warm ions. While this is a valid assumption for a lowbeta plasma regime such as encountered in this event, warm plasma effects can significantly modify dispersion surfaces in general (Silin et al., 2011). Extraneous magnetic pulsations over the same time period as the wave can contaminate the minimum variance analysis and introduce uncertainty in wave vector direction, which in turn may result in an erroneous $\theta_{k R}$ and an uncertainty about the wavenumber. The magnetic field strength varies slightly for the duration of the event, while the Morlet wavelet spectral plot makes precise identification of the cut-off frequency problematic. The above errors in turn cause uncertainty in calculating plasma composition. The plasma electron resonant frequency is out of range of the WHISPER instrument, meaning that the total electron density has to be computed indirectly (Pickett et al., 2010, and references therein). Despite these limitations the results fit the theoretical data fairly well. Estimated inaccuracies are represented by error bars in Fig. 6 .

The assumption that EMIC emissions cannot propagate in the stop band only holds for low plasma beta regimes. Work by Silin et al. (2011) suggests that, under high-beta conditions, EMIC waves can be excited anywhere in the stop band. The cold plasma approximation then breaks down, and the dispersion should be calculated using the full dispersion relation. Such an estimation requires a detailed knowledge of not only the densities of each species but also knowledge of the full distribution function of each of the species. The method described in this study can be used to derive the dispersion function empirically for any conditions from observations, providing the wave vector can be determined. As such it can 
be a useful tool to validate theoretical dispersion functions under unusual plasma regimes.

For the first time multiple satellite measurements have been used in conjunction with the phase differencing method to compare directly the experimental and the theoretical dispersion relation of EMIC waves just below the $\mathrm{H}^{+}$gyrofrequency. The evolution of normalised wave frequency with wave vector is similar to the analytical results obtained under the linear dispersion theory for $L$ mode waves. Some discrepancies are probably due to the difficulty in ascertaining exact ion ratios.

Acknowledgements. We acknowledge the ESA Cluster Active Archive and the STAFF, FGM, WHISPER and CIS teams for supplying the Cluster data. We acknowledge discussions with Richard Horne, Richard Boynton and Andrew Dimmock. The research leading to these results was funded by STFC. Y. Y. Shprits would like to acknowledge UC Lab Fee Grant and NASA grants NNX10AK99G; NNX10AK99G

Topical Editor I. A. Daglis thanks two anonymous referees for their help in evaluating this paper.

\section{References}

Anderson, B. J., Erlandson, R. E., and Zanetti, L. J.: A statistical study of Pc 1-2 magnetic pulsations in the equatorial magnetosphere: 1. Equatorial occurrence distributions, J. Geophys. Res.: Space Physics, 97, 3075-3088, doi:10.1029/91JA02706, 1992.

Baker, D.: Solar wind-magnetosphere drivers of space weather, J. Atmos. Terr. Phys., 58, 1509-1526, doi:10.1016/00219169(96)00006-2, 1996.

Balikhin, M. A. and Gedalin, M. E.: Comparative analysis of different methods for distinguishing temporal and spatial variations, in: Proc. of START Conf., Aussois, France, vol. ESA WPP 047, pp. 183-187, 1993.

Balikhin, M. A., de Wit, T. D., Alleyne, H. S. C. K., Woolliscroft, L. J. C., Walker, S. N., Krasnosel'skikh, V., Mier-Jedrzejeowicz, W. A. C., and Baumjohann, W.: Experimental determination of the dispersion of waves observed upstream of a quasi-perpendicular shock, Geophys. Res. Lett., 24, 787-790, 1997a.

Balikhin, M. A., Woolliscroft, L. J. C., Alleyne, H. St. C., Dunlop, M., and Gedalin, M. A.: Determination of the dispersion of low frequency waves downstream of a quasiperpendicular collisionless shock, Ann. Geophys., 15, 143-151, doi:10.1007/s00585997-0143-x, 1997b.

Balikhin, M., Bates, I., and Dunlop, M.: Minimum Variance Free Identification of the Composition of Space Plasma Turbulence and Nonlinear Processes in it, Proceedings of the ClusterII Workshop Multiscale/Multipoint Plasma Measurements, SP449, 287-290, 2000.

Balogh, A., Carr, C. M., Acuña, M. H., Dunlop, M. W., Beek, T. J., Brown, P., Fornacon, K.-H., Georgescu, E., Glassmeier, K.H., Harris, J., Musmann, G., Oddy, T., and Schwingenschuh, K.: The Cluster Magnetic Field Investigation: overview of in-flight performance and initial results, Ann. Geophys., 19, 1207-1217, doi:10.5194/angeo-19-1207-2001, 2001.
Broughton, M. C., Engebretson, M. J., Glassmeier, K.-H., Narita, Y., Keiling, A., Fornaçon, K.-H., Parks, G. K., and Rème, H.: Ultra-low-frequency waves and associated wave vectors observed in the plasma sheet boundary layer by Cluster, J. Geophys. Res.: Space Physics, 113, A12217, doi:10.1029/2008JA013366, 2008.

Chen, Y., Friedel, R. H. W., and Reeves, G. D.: Phase space density distributions of energetic electrons in the outer radiation belt during two Geospace Environment Modeling Inner Magnetosphere/Storms selected storms, J. Geophys. Res., 111, A11S04, doi:10.1029/2006JA011703, 2006.

Chisham, G., Schwartz, S., Balikhin, M., and Dunlop, M.: AMPTE observations of mirror mode waves in the magnetosheath: Wavevector determination, J. Geophys. Res., 104, 437-447, 1999.

Cornilleau-Wehrlin, N., Chanteur, G., Perraut, S., Rezeau, L., Robert, P., Roux, A., de Villedary, C., Canu, P., Maksimovic, M., de Conchy, Y., Hubert, D., Lacombe, C., Lefeuvre, F., Parrot, M., Pinçon, J. L., Décréau, P. M. E., Harvey, C. C., Louarn, Ph., Santolik, O., Alleyne, H. St. C., Roth, M., Chust, T., Le Contel, O., and STAFF team: First results obtained by the Cluster STAFF experiment, Ann. Geophys., 21, 437-456, doi:10.5194/angeo-21437-2003, 2003.

Décréau, P., Fergeau, P., Krannosels'kikh, V., Lèvêque, Martin, P., Randriamboarison, O., Senè, F., Trotignon, J., Canu, P., Mögensen, P., and Investigators, W.: WHISPER, A Resonance Sounder And Wave Analyser: Performances and Perspectives for the Cluster Mission, Space Sci. Rev., 79, 157-193, 1997.

Dudok de Wit, T., Krasnosel'skikh, V. V., Bale, S. D., Dunlop, M. W., Lühr, H., Schwartz, S. J., and Woolliscroft, L. J. C.: Determination of dispersion relations in quasi-stationary plasma turbulence using dual satellite data, Geophys. Res. Lett., 22, 2653 2656, doi:10.1029/95GL02543, 1995.

Elkington, S. R., Hudson, M. K., and Chan, A. A.: Resonant acceleration and diffusion of outer zone electrons in an asymmetric geomagnetic field, J. Geophys. Res., 108, 1116, doi:10.1029/2001JA009202, 2003.

Fälthammar, C.-G.: Effects of time-dependent electric fields on geomagnetically trapped radiation, J. Geophys. Res., 70, 2503-2516, doi:10.1029/JZ070i011p02503, 1965.

Fowler, R. A., Kotick, B. J., and Elliott, R. D.: Polarization Analysis of Natural and Artificially Induced Geomagnetic Micropulsations, J. Geophys. Res., 72, 2871-2883, 1967.

Fraser, B. and Nguyen, T.: Is the plasmapause a preferred source region of electromagnetic ion cyclotron waves in the magnetosphere?, Journal of Atmospheric and Solar-Terrestrial Physics, 63, 1225-1247, doi:10.1016/S1364-6826(00)00225-X, 2001.

Fraser, B. J., Samson, J. C., Hu, Y. D., McPherron, R. L., and Russell, C. T.: Electromagnetic ion cyclotron waves observed near the oxygen cyclotron frequency by ISEE 1 and 2, J. Geophys. Res., 97, 3063-3074, doi:10.1029/91JA02447, 1992.

Fraser, B. J., Singer, H. J., Hughes, W. J., Wygant, J. R., Anderson, R. R., and Hu, Y. D.: CRRES Poynting vector observations of electromagnetic ion cyclotron waves near the plasmapause, J. Geophys. Res., 101, 15331-15343, doi:10.1029/95JA03480, 1996.

Fraser, B. J., Grew, R. S., Morley, S. K., Green, J. C., Singer, H. J., Loto'aniu, T. M., and Thomsen, M. F.: Storm time observations of electromagnetic ion cyclotron waves at geosyn- 
chronous orbit: GOES results, J. Geophys. Res., 115, A05208, doi:10.1029/2009JA014516, 2010.

Friedel, R., Reeves, G., and Obara, T.: Relativistic electron dynamics in the inner magnetosphere - a review, J. Atmos. Solar-Terr. Phys., 64, 265-282, doi:10.1016/S1364-6826(01)00088-8, 2002.

Grison, B., Santolik, O., Cornilleau-Wehrlin, N., Masson, A., Engebretson, M. J., Pickett, J. S., Omura, Y., Robert, P., and Nomura, R.: EMIC triggered chorus emissions in Cluster data, J. Geophys. Res., 118, 1159-1169, doi:10.1002/jgra.50178, 2013.

Horne, R. B. and Thorne, R. M.: Ion cyclotron absorption at the second harmonic of the oxygen gyrofrequency, Geophys. Res. Lett., 17, 2225-2228, 1990.

Horne, R. B. and Thorne, R. M.: Convective Instabilities of Electromagnetic Ion Cyclotron Waves in the Outer Magnetosphere, J. Geophys. Res., 99, 17259-17273, 1994.

Kellogg, P. J.: Van Allen Radiation of Solar Origin, Nature, 183, 1295-1297, 1959.

Li, W., Shprits, Y. Y., and Thorne, R. M.: Dynamic evolution of energetic outer zone electrons due to wave-particle interactions during storms, J. Geophys. Res., 112, A10220, doi:10.1029/2007JA012368, 2007.

Li, X., Baker, D. N., Kanekal, S. G., Looper, M., and Temerin, M.: Long term measurements of radiation belts by SAMPEX and their variations, Geophys. Res. Lett., 28, 3827-3830, 2001.

Lyons, L. R. and Thorne, R. M.: Equilibrium structure of radiation belt electrons, J. Geophys. Res., 78, 2142-2149, doi:10.1029/JA078i013p02142, 1973.

Lyons, L. R., Thorne, R. M., and Kennel, C. F.: Pitch-angle diffusion of radiation belt electrons within the plasmasphere, J. Geophys. Res., 77, 3455-3474, doi:10.1029/JA077i019p03455, 1972.

Meredith, N. P., Thorne, R. M., Horne, R. B., Summers, D., Fraser, B. J., and Anderson, R. R.: Statistical analysis of relativistic electron energies for cyclotron resonance with EMIC waves observed on CRRES, J. Geophys. Res., 108, 1250, doi:10.1029/2002JA009700, 2003.

Omura, Y., Pickett, J., Grison, B., Santolik, O., Dandouras, I., Engebretson, M., Dècrèau, P. M. E., and Masson, A.: Theory and observation of electromagnetic ion cyclotron triggered emissions in the magnetosphere, J. Geophys. Res., 115, A07234, doi:10.1029/2010JA015300, 2010.

Pickett, J. S., Grison, B., Omura, Y., Engebretson, M. J., Dandouras, I., Masson, A., Adrian, M. L., Santolík, O., Décréau, P. M. E., Cornilleau-Wehrlin, N., and Constantinescu, D.: Cluster observations of EMIC triggered emissions in association with Pc1 waves near Earth's plasmapause, Geophys. Res. Lett., 37, L09104, doi:10.1029/2010GL042648, 2010.

Reeves, G. D., McAdams, K. L., Friedel, R. H. W., and O'Brien, T. P.: Acceleration and loss of relativistic electrons during geomagnetic storms, Geophys. Res. Lett., 30, 1529, doi:10.1029/2002GL016513, 2003.

Rème, H., Aoustin, C., Bosqued, J. M., Dandouras, I., Lavraud, B., Sauvaud, J. A., Barthe, A., Bouyssou, J., Camus, Th., Coeur-Joly, O., Cros, A., Cuvilo, J., Ducay, F., Garbarowitz, Y., Medale, J. L., Penou, E., Perrier, H., Romefort, D., Rouzaud, J., Vallat, C., Alcaydé, D., Jacquey, C., Mazelle, C., d'Uston, C., Möbius, E., Kistler, L. M., Crocker, K., Granoff, M., Mouikis, C., Popecki, M., Vosbury, M., Klecker, B., Hovestadt, D., Kucharek, H., Kuenneth, E., Paschmann, G., Scholer, M., Sckopke, N., Seiden- schwang, E., Carlson, C. W., Curtis, D. W., Ingraham, C., Lin, R. P., McFadden, J. P., Parks, G. K., Phan, T., Formisano, V., Amata, E., Bavassano-Cattaneo, M. B., Baldetti, P., Bruno, R., Chionchio, G., Di Lellis, A., Marcucci, M. F., Pallocchia, G., Korth, A., Daly, P. W., Graeve, B., Rosenbauer, H., Vasyliunas, V., McCarthy, M., Wilber, M., Eliasson, L., Lundin, R., Olsen, S., Shelley, E. G., Fuselier, S., Ghielmetti, A. G., Lennartsson, W., Escoubet, C. P., Balsiger, H., Friedel, R., Cao, J.-B., Kovrazhkin, R. A., Papamastorakis, I., Pellat, R., Scudder, J., and Sonnerup, B.: First multispacecraft ion measurements in and near the Earth's magnetosphere with the identical Cluster ion spectrometry (CIS) experiment, Ann. Geophys., 19, 1303-1354, doi:10.5194/angeo19-1303-2001, 2001.

Selesnick, R. S., Blake, J. B., and Mewaldt, R. A.: Atmospheric losses of radiation belt electrons, J. Geophys. Res., 108, 1468, doi:10.1029/2003JA010160, 2003.

Shoji, M., Omura, Y., Grison, B., Pickett, J., Dandouras, I., and Engebretson, M.: Electromagnetic ion cyclotron waves in the helium branch induced by multiple electromagnetic ion cyclotron triggered emissions, Geophys. Res. Lett., 38, L17102, doi:10.1029/2011GL048427, 2011.

Shprits, Y., Kondrashov, D., Chen, Y., Thorne, R., Ghil, M., Friedel, R., and Reeves, G.: Reanalysis of relativistic radiation belt electron fluxes using CRRES satellite data, a radial diffusion model, and a Kalman filter, J. Geophys. Res., 112, A12216, doi:10.1029/2007JA012579, 2007.

Shprits, Y. Y., Elkington, S. R., Meredith, N. P., and Subbotin, D. A.: Review of modeling of losses and sources of relativistic electrons in the outer radiation belt I: Radial transport, J. Atmos. SolarTerr. Phys., 70, 1679-1693, doi:10.1016/j.jastp.2008.06.008, 2008a.

Shprits, Y. Y., Subbotin, D. A., Meredith, N. P., and Elkington, S. R.: Review of modeling of losses and sources of relativistic electrons in the outer radiation belt II: Local acceleration and loss, J. Atmos. Solar-Terr. Phys., 70, 1694-1713, doi:10.1016/j.jastp.2008.06.014, 2008b.

Silin, I., Mann, I. R., Sydora, R. D., Summers, D., and Mace, R. L.: Warm plasma effects on electromagnetic ion cyclotron wave $\mathrm{MeV}$ electron interactions in the magnetosphere, J. Geophys. Res., 116, A05215, doi:10.1029/2010JA016398, 2011.

Smith, R. L. and Brice, N.: Propagation in Multicomponent Plasmas, J. Geophys. Res., 69, 5029-5040, 1964.

Sonnerup, B. U. Ö. and Cahill, L. J., J.: Magnetopause Structure and Attitude from Explorer 12 Observations, J. Geophys. Res., 72, 171-183, 1967.

Stix, T. H.: The Theory of Plasma Waves, Mc-Graw Hill, New York, 1962.

Summers, D. and Thorne, R. M.: Relativistic electron pitchangle scattering by electromagnetic ion cyclotron waves during geomagnetic storms, J. Geophys. Res., 108, 1143, doi:10.1029/2002JA009489, 2003.

Summers, D., Ma, C., Meredith, N. P., Horne, R. B., Thorne, R. M., Heynderickx, D., and Anderson, R. R.: Model of the energization of outer-zone electrons by whistler-mode chorus during the October 9, 1990 geomagnetic storm, Geophys. Res. Lett., 29, 27-1-27-4, doi:10.1029/2002GL016039, 2002.

Swanson, D. G.: Plasma Waves, Institute of Physics Publishing, Bristol and Philadelphia, 1989. 
Thorne, R. M.: Radiation belt dynamics: The importance of wave-particle interactions, Geophys. Res. Lett., 37, L22107, doi:10.1029/2010GL044990, 2010.

Thorne, R. M., Shprits, Y. Y., Meredith, N. P., Horne, R. B., Li, W., and Lyons, L. R.: Refilling of the slot region between the inner and outer electron radiation belts during geomagnetic storms, J. Geophys. Res., 112, A06203, doi:10.1029/2006JA012176, 2007.

Tverskoy, B. A.: Dynamics of the radiation belts of the Earth, 2, Geomagn. Aeron., Engl. Transl., 4, 351-366, 1964.

Tverskoy, B. A.: Transport and acceleration of charged particles in the Earth's magnetosphere, Geomagn. Aeron., Engl. Transl., 5, 617-628, 1965.

Walker, S. N., Sahraoui, F., Balikhin, M. A., Belmont, G., Pinçon, J. L., Rezeau, L., Alleyne, H., Cornilleau-Wehrlin, N., and André, M.: A comparison of wave mode identification techniques, Ann. Geophys., 22, 3021-3032, doi:10.5194/angeo-223021-2004, 2004.
Woolliscroft, L., Alleyne, H., Dunford, C., Sumner, A., Thompson, J., Walker, S., Yearby, K., Buckley, A., Chapman, S., Gough, M., Balikhin, M., Burgess, D., CornilleauWehrlin, N., Roux, A., Decreau, P., Krasnoselskikh, V., Lefeuvre, F., Parrot, M., Egeland, A., Gedalin, M., Gurnett, D., Gustafsson, G., Holmgren, G., Harvey, C., Horne, R., Iversen, I., Kofman, W., Koons, H., LaBelle, J., Mozer, F., and Reznikov, A.: The digital wave-processing experiment on Cluster, Space Sci. Rev., 79, 209-231, 1997.

Young, D. T., Perraut, S., Roux, A., de Villedary, C., Gendrin, R., Korth, A., Kremser, G., and Jones, D.: Wave-particle interactions near $\Omega_{\mathrm{He}^{+}}$observed on geos 1 and 2, 1. propagation of ion cyclotron waves in $\mathrm{He}^{+}$-rich plasma, J. Geophys. Res., 86, 6755$6772,1981$. 